\title{
Polyhedra with finite fundamental group dominate finitely many different homotopy types
}

by

\author{
Danuta Kołodziejczyk (Warszawa)
}

\begin{abstract}
In $1968 \mathrm{~K}$. Borsuk asked: Does every polyhedron dominate only finitely many different shapes? In this question the notion of shape can be replaced by the notion of homotopy type. We showed earlier that the answer to the Borsuk question is no. However, in a previous paper we proved that every simply connected polyhedron dominates only finitely many different homotopy types (equivalently, shapes). Here we prove that the same is true for polyhedra with finite fundamental group.
\end{abstract}

Introduction. By a polyhedron we mean, as usual, a finite one. In this paper, all polyhedra and $C W$-complexes are assumed to be connected (for convenience). The exposition of basic notions and results of shape theory can be found in [DS], [MS] or [B3].

In 1968, at the Topological Conference in Herceg-Novi, K. Borsuk asked:

Does every polyhedron dominate only finitely many different shapes? (see also [B2], and [B1], where an equivalent question was stated for FANRs).

Let us note that this question is equivalent to the following:

Does every polyhedron dominate only finitely many different homotopy types?

Indeed, each space homotopy dominated by a polyhedron has the homotopy type of a $C W$-complex, not necessarily finite (J. H. C. Whitehead; see also [Wa]). Moreover, as a consequence of the known results of shape theory (see [HaHe], [DS, Theorem 2.2.6]), there is a 1-1 functorial correspondence between the shapes of compacta shape dominated by a given polyhedron and the homotopy types of $C W$-complexes homotopy dominated by it (in

2000 Mathematics Subject Classification: 55P55, 55P15.

Key words and phrases: polyhedron, $C W$-complex, homotopy type, homotopy domination, shape, shape domination. 
both pointed and unpointed cases). Furthermore, in both pointed and unpointed cases the answer to the Borsuk question is the same (see [HaHe], [D, Theorem 5.1]).

From the earlier result of $[\mathrm{Ma}]$ one can deduce that each polyhedron dominates only a countable number of different shapes (see also [Ho], [MP]).

We showed ([K3]) that, generally, the answer to the Borsuk question is no: there exist polyhedra (even of dimension 2) homotopy dominating infinitely many polyhedra of different homotopy types (see also [K4]). In [K1] we proved that this phenomenon is not rare: for every non-abelian poly-ZZ-group $G$, there exists a polyhedron $P$ with $\pi_{1}(P) \cong G$ dominating infinitely many polyhedra of different homotopy types. It should be noted that, by the classical results of shape theory, every 1-dimensional polyhedron dominates only finitely many different shapes (see [B3, Theorem 7.1, p. 221], and $[\operatorname{Tr}])$.

On the other hand, in [K2], applying the results of localization theory in the homotopy category of $C W$-complexes, we proved that every simply connected polyhedron dominates only finitely many different homotopy types (or equivalently, shapes).

Here we prove, in another way, that every polyhedron with finite fundamental group dominates only finitely many different homotopy types (the method of [K2] does not apply in this case).

We will consider here dominations of a polyhedron in the category of $C W$-complexes and homotopy classes of cellular maps between them, and also the pointed version of the question.

Main results. Let $\mathcal{M}_{n}(K)$ denote the set of all matrices $n \times n$ over a field $K$.

DeFINITION 1. By an idempotent in a category $\mathcal{C}$ we mean a morphism $k$ such that $k \circ k=k$ in $\mathcal{C}$.

The lemma below, due to M. S. Putcha, can be deduced from the Hilbert Basis Theorem (for this theorem, see for example [Pu1, Ch. 2]).

Lemma ([Pu2, Lemma 1.6, p. 459]). Let $\mathcal{E}$ be an infinite set of idempotents in $\mathcal{M}_{n}(K)$ of rank $r$, where $K$ is a given algebraically closed field. Then there exist two idempotents $E, F \in \mathcal{E}$ such that $E \neq F$ and $\operatorname{rank}(E F)=$ $\operatorname{rank}(F E)=r$.

As an immediate corollary we obtain (cf. [Pu2, Theorem 1.7] and [Re, Proposition 2.2]):

Corollary. Let $K$ be an algebraically closed field. Then any given subset $\mathcal{E}$ of idempotents in $\mathcal{M}_{n}(K)$ of rank $r$ is partitioned into finitely many classes such that $E$ and $F$ belong to the same class if and only if there exists 
a finite set of idempotents $E_{0}=E, E_{1}, \ldots, E_{m}=F$, where $E_{j} \in \mathcal{E}$ for each $j=0, \ldots, m$, and $\operatorname{rank}\left(E_{j-1} E_{j}\right)=\operatorname{rank}\left(E_{j} E_{j-1}\right)=r$ for $j=1, \ldots, m$.

REMARK. Since every field has an algebraic closure, in this corollary the assumption that $K$ is algebraically closed can be omitted.

For a polyhedron $P$, let $X \leq P$ denote that $X$ is homotopy dominated by $P$. Writing this we will have in mind a fixed domination $d_{X}: P \rightarrow X$ of $P$ over $X$, and a fixed inverse map $u_{X}: X \rightarrow P$ (i.e. $\left.d_{X} u_{X} \simeq \operatorname{id}_{X}\right)$.

It is easily seen that the map $k_{X}=u_{X} d_{X}: P \rightarrow P$ is an idempotent in the homotopy category of $C W$-complexes and homotopy classes of maps between them.

Recall that $\mathbb{Z} / p$ denotes the integers modulo $p \in \mathbb{P}$, where $\mathbb{P}$ is the set of all primes.

Definition 2. (i) A map $f: X \rightarrow Y$ between $C W$-complexes $X$ and $Y$ is said to be a homology equivalence if it induces an isomorphism of the integral homology groups, $H_{i}(f): H_{i}(X ; \mathbb{Z}) \rightarrow H_{i}(Y ; \mathbb{Z})$, for all $i \in \mathbb{N}$ (cf. [WhG, pp. 181-182]).

(ii) Similarly, $f$ will be called a $\mathbb{Q}$-homology equivalence if it induces an isomorphism of the homology groups with coefficients $\mathbb{Q}, H_{i, \mathbb{Q}}(f): H_{i}(X ; \mathbb{Q})$ $\rightarrow H_{i}(Y ; \mathbb{Q})$, for all $i \in \mathbb{N}$.

(iii) Given a prime $p \in \mathbb{P}, f$ will be said to be a $\mathbb{Z} / p$-homology equivalence if it induces an isomorphism of the homology groups with coefficients $\mathbb{Z} / p$, $H_{i, p}(f): H_{i}(X ; \mathbb{Z} / p) \rightarrow H_{i}(Y ; \mathbb{Z} / p)$, for all $i \in \mathbb{N}$.

From now on "dominated" will always mean "homotopy dominated".

Let us prove the following

Lemma 1. Let $\mathcal{W}$ be a subclass of the class of all $C W$-complexes dominated by a polyhedron $P$. Then $\mathcal{W}$ can be partitioned into finitely many classes such that if $X$ and $Y$ belong to the same class, then there exists a finite sequence $\left\{X_{j}\right\}_{j=0}^{m}$, where $X_{0}=X, X_{m}=Y, X_{j} \in \mathcal{W}$, such that the map $g_{X Y}=d_{Y} k_{X_{m-1}} k_{X_{m-2}} \ldots k_{X_{2}} k_{X_{1}} u_{X}: X \rightarrow Y$ is a $\mathbb{Q}$-homology equivalence.

Proof. For every $i \in \mathbb{N}$, consider

$$
H_{i}(P ; \mathbb{Q}) \cong H_{i}(P) \otimes \mathbb{Q} \cong \underbrace{\mathbb{Q} \oplus \mathbb{Q} \oplus \ldots \oplus \mathbb{Q}}_{l_{i}},
$$

an $l_{i}$-dimensional vector space over $\mathbb{Q}$, where $l_{i}=\beta_{i}(P)$.

Any homomorphism of $H_{i}(P ; \mathbb{Q})$ can be represented by some matrix in $\mathcal{M}_{l_{i}}(\mathbb{Q})$, and there is a 1-1 correspondence between idempotent homomorphisms of $H_{i}(P ; \mathbb{Q})$ and idempotent matrices in $\mathcal{M}_{l_{i}}(\mathbb{Q})$.

Given $X \in \mathcal{W}$, let $M_{i}(X) \in \mathcal{M}_{l_{i}}(\mathbb{Q})$ be the matrix of the homomorphism $H_{i, \mathbb{Q}}\left(k_{X}\right): H_{i}(P ; \mathbb{Q}) \rightarrow H_{i}(P ; \mathbb{Q})$ induced by the idempotent $k_{X}: P \rightarrow P$. 
Consider the $\mathbb{Q}$-vector space $V=\bigoplus_{i=1}^{n} H_{i}(P ; \mathbb{Q})$, where $n=\operatorname{dim} P$. Denote by $M(X) \in \mathcal{M}_{l}(\mathbb{Q})$, where $l=\sum_{i=1}^{n} l_{i}$, the matrix of the homomorphism $k_{X *}=\bigoplus_{i=1}^{n} H_{i, \mathbb{Q}}\left(k_{X}\right): V \rightarrow V$. Then $M(X)$ is composed of the matrices $M_{i}(X)$ as follows:

$$
M(X)=\left[\begin{array}{ccc}
M_{1}(X) & & \text { zeroes } \\
& \ddots & \\
\text { zeroes } & & M_{n}(X)
\end{array}\right] .
$$

Since all the matrices $M_{i}(X)$ are idempotent, so also is $M(X)$. Moreover, $r(X)=\sum_{i=1}^{n} r_{i}(X)$, where $r(X)=\operatorname{rank} M(X)$ and $r_{i}(X)=\operatorname{rank} M_{i}(X)$ for $i=1, \ldots, n$.

To prove the lemma, in the first step we partition $\mathcal{W}$ into finitely many classes by putting two $C W$-complexes in the same class if the collections $\left(r_{1}(X), \ldots, r_{n}(X)\right)$ are the same.

Fix any class $\mathcal{W}^{\prime}$ of this partition. Let $\mathcal{E}$ be the class of all matrices $M(X) \in \mathcal{M}_{l}(\mathbb{Q})$ for $X \in \mathcal{W}^{\prime}$ (clearly of a fixed rank $r=\sum_{i=1}^{n} r_{i}(X)$ ). Apply the Corollary to divide $\mathcal{E}$ into finitely many classes such that if $M(X)$ and $M(Y)$ belong to the same class, then there exists a finite sequence $\left\{M_{j}\right\}_{j=0}^{m}$, where $m \geq 1$, of matrices in $\mathcal{E}$ with $M_{0}=M(X), M_{m}=M(Y)$, $\operatorname{rank} M_{j-1}=\operatorname{rank} M_{j}=\operatorname{rank}\left(M_{j-1} M_{j}\right)=\operatorname{rank}\left(M_{j} M_{j-1}\right)=r$, for each $j=1, \ldots, m$.

This generates a partition of $\mathcal{W}^{\prime}$ into finitely many classes such that $X$ and $Y$ belong to the same class if and only if $M(X)$ and $M(Y)$ belong to the same class of the partition of $\mathcal{E}$ as above.

Thus, for $X$ and $Y$ in the same class, there exists a sequence $\left\{X_{j}\right\}_{j=0}^{m}$, where $m \geq 1$, of $C W$-complexes $X_{j} \in \mathcal{W}^{\prime}$ such that $X_{0}=X, X_{m}=Y$, and

$$
\begin{aligned}
\operatorname{rank} M\left(X_{j-1}\right) & =\operatorname{rank} M\left(X_{j}\right)=\operatorname{rank} M\left(X_{j-1}\right) M\left(X_{j}\right) \\
& =\operatorname{rank} M\left(X_{j}\right) M\left(X_{j-1}\right)
\end{aligned}
$$

for $j=1, \ldots, m$. It follows that

$$
\begin{aligned}
\operatorname{rank} M_{i}\left(X_{j-1}\right) & =\operatorname{rank} M_{i}\left(X_{j}\right)=\operatorname{rank} M_{i}\left(X_{j-1}\right) M_{i}\left(X_{j}\right) \\
& =\operatorname{rank} M_{i}\left(X_{j}\right) M_{i}\left(X_{j-1}\right)
\end{aligned}
$$

for each $i=1, \ldots, n$ and $j=1, \ldots, m$.

To see this, note that $r\left(X_{j}\right)=\sum_{i=1}^{n} r_{i}\left(X_{j}\right)$, and for any two matrices $A$ and $B$ we have $\operatorname{rank} A B \leq \operatorname{rank} A$ and $\operatorname{rank} A B \leq \operatorname{rank} B$.

We claim that the map $g_{X Y}=d_{Y} k_{X_{m-1}} k_{X_{m-2}} \ldots k_{X_{2}} k_{X_{1}} u_{X}: X \rightarrow Y$ is a $\mathbb{Q}$-homology equivalence.

Indeed, by the well-known facts from linear algebra, the condition $(*)$ implies that $H_{i, \mathbb{Q}}\left(k_{X_{j}}\right) \mid \operatorname{im} H_{i, \mathbb{Q}}\left(k_{X_{j-1}}\right)$ is an isomorphism onto im $H_{i, \mathbb{Q}}\left(k_{X_{j}}\right)$ for $1 \leq j \leq m$. 
Clearly $u_{X}: X \rightarrow P$ induces an isomorphism $H_{i, \mathbb{Q}}\left(u_{X}\right)$ of $H_{i}(X ; \mathbb{Q})$ onto $\operatorname{im} H_{i, \mathbb{Q}}\left(u_{X}\right)=\operatorname{im} H_{i, \mathbb{Q}}\left(k_{X}\right)$.

Moreover, $H_{i, \mathbb{Q}}\left(k_{Y}\right) \mid \operatorname{im} H_{i, \mathbb{Q}}\left(k_{X_{m-1}}\right)$ is an isomorphism onto

$$
\operatorname{im} H_{i, \mathbb{Q}}\left(k_{Y}\right)=\operatorname{im} H_{i, \mathbb{Q}}\left(u_{Y}\right),
$$

and $H_{i, \mathbb{Q}}\left(u_{Y}\right)$ is an isomorphism of $H_{i}(Y ; \mathbb{Q})$ onto $\operatorname{im} H_{i, \mathbb{Q}}\left(u_{Y}\right)$, hence $H_{i, \mathbb{Q}}\left(d_{Y}\right) \mid \operatorname{im} H_{i, \mathbb{Q}}\left(k_{X_{m-1}}\right)$ is an isomorphism onto $H_{i}(Y, \mathbb{Q})$. This completes the proof of the claim and of the lemma.

Definition 3 (cf. [HMR, Definition 1.3, p. 5]). Let $\mathbb{P}^{\prime} \subseteq \mathbb{P}$ be a subset of primes and $\mathbb{P}^{\prime \prime} \subseteq \mathbb{P}$ the complementary subset of primes. If an integer $n$ is a product of primes belonging to $\mathbb{P}^{\prime \prime}$, we write $n \in \mathbb{P}^{\prime \prime}$.

We say that a homomorphism $g: G \rightarrow K$ of finitely generated abelian groups is

(i) $\mathbb{P}^{\prime}$-injective if ker $g$ consists of $\mathbb{P}^{\prime \prime}$-torsion elements;

(ii) $\mathbb{P}^{\prime}$-surjective if, given any $y \in K$, there exists an integer $n \in \mathbb{P}^{\prime \prime}$ such that $y^{n} \in \operatorname{im} g$;

(iii) a $\mathbb{P}^{\prime}$-isomorphism if it is both $\mathbb{P}^{\prime}$-injective and $\mathbb{P}^{\prime}$-surjective.

We will apply this definition to $\mathbb{P}^{\prime}=\{p\}$; then $\mathbb{P}^{\prime}$-isomorphism will be called p-isomorphism.

Lemma 2. Let $X$ and $Y$ be $C W$-complexes with finitely generated integral homology groups and let $f: X \rightarrow Y$ be a $\mathbb{Q}$-homology equivalence. Then $f$ is also a $\mathbb{Z} / p$-homology equivalence for all $p \in S$, where $S=\{p \in \mathbb{P} \mid$ $p$ divides no torsion coefficient of the groups $H_{i}(X ; \mathbb{Z})$ and $H_{i}(Y ; \mathbb{Z})$ for $i=1,2, \ldots\}$.

Proof. Let $M_{f} \simeq Y$ be the mapping cylinder of $f$. Since $f: X \rightarrow Y$ is a $\mathbb{Q}$-homology equivalence, we have $H_{i}\left(M_{f}, X ; \mathbb{Q}\right)=0$ for all $i \in \mathbb{N}$. By the Universal Coefficient Theorem, $H_{i}\left(M_{f}, X ; \mathbb{Q}\right) \cong H_{i}\left(M_{f}, X ; \mathbb{Z}\right) \otimes \mathbb{Q}$, therefore $H_{i}\left(M_{f}, X ; \mathbb{Z}\right)$ is a finite abelian group for all $i \in \mathbb{N}$.

We will use the following assertion:

(*) Let $f: X \rightarrow Y$, where $X$ and $Y$ are $C W$-complexes with all integral homology groups finitely generated. If for a prime $p \in \mathbb{P}, H_{i}(f)$ : $H_{i}(X ; \mathbb{Z}) \rightarrow H_{i}(Y ; \mathbb{Z})$ is a $p$-isomorphism for all $i \in \mathbb{N}$, then $f$ is a $\mathbb{Z} / p$-homology equivalence.

This is a consequence of the Universal Coefficient Theorem and a part of [HMR, Proposition 1.8] which states that for two abelian groups $A$ and $B$, a $p$-isomorphism $\Phi: A \rightarrow B$ induces isomorphisms $\Phi \otimes \mathbb{Z} / p$ and $\operatorname{Tor}(\Phi, \mathbb{Z} / p)$.

Let $p \in S$. Using the exact sequence

$$
\begin{aligned}
\ldots \rightarrow H_{i}(X ; \mathbb{Z}) \rightarrow H_{i}\left(M_{f} ; \mathbb{Z}\right) & \rightarrow H_{i}\left(M_{f}, X ; \mathbb{Z}\right) \\
& \rightarrow H_{i-1}(X ; \mathbb{Z}) \rightarrow H_{i-1}\left(M_{f} ; \mathbb{Z}\right) \rightarrow \ldots
\end{aligned}
$$


one can check that $f$ induces a $p$-isomorphism $H_{i}(f): H_{i}(X ; \mathbb{Z}) \rightarrow H_{i}(Y ; \mathbb{Z})$ for all $i \in \mathbb{N}$. By $(*)$, this completes the proof.

Lemma 3. Let $X$ and $Y$ be $C W$-complexes with finitely generated integral homology groups and let $f: X \rightarrow Y$ be a $\mathbb{Q}$-homology equivalence and $\mathbb{Z} / p$-homology equivalence for each $p \in \mathbb{P}$. Then $f$ is a homology equivalence.

Proof. By the argument as in the proof of Lemma $2, H_{i}\left(M_{f}, X ; \mathbb{Z}\right)$ is a finite abelian group for all $i \in \mathbb{N}$. The Universal Coefficient Theorem states that, for each $p \in \mathbb{P}$ and $i \in \mathbb{N}$,

$$
H_{i}\left(M_{f}, X ; \mathbb{Z} / p\right) \cong\left(H_{i}\left(M_{f}, X ; \mathbb{Z}\right) \otimes \mathbb{Z} / p\right) \oplus \operatorname{Tor}\left(\mathbb{Z} / p ; H_{i-1}\left(M_{f}, X ; \mathbb{Z}\right)\right) .
$$

Since $f: X \rightarrow Y$ is a $\mathbb{Z} / p$-homology equivalence for all $p \in \mathbb{P}$, we have $H_{i}\left(M_{f}, X ; \mathbb{Z} / p\right)=0$ for each $i \in \mathbb{N}$ and $p \in \mathbb{P}$. Therefore all $H_{i}\left(M_{f}, X ; \mathbb{Z}\right)$ $=0$. Indeed, $H_{i}\left(M_{f}, X ; \mathbb{Z}\right) \otimes \mathbb{Z} / p \neq 0$ for each prime $p \in \mathbb{P}$ dividing some torsion coefficient of $H_{i}\left(M_{f}, X ; \mathbb{Z}\right)$. This finishes the proof.

Lemma 4. Let $P$ be a polyhedron. Any subclass $\mathcal{W}$ of the class of all $C W$-complexes dominated by $P$ can be partitioned into finitely many classes such that if $X$ and $Y$ belong to the same class, then there exists a finite sequence $\left\{X_{j}\right\}_{j=0}^{m}$, where $X_{0}=X, X_{m}=Y, X_{j} \in \mathcal{W}$, such that the map $g_{X Y}=d_{Y} k_{X_{m-1}} k_{X_{m-2}} \ldots k_{X_{2}} k_{X_{1}} u_{X}: X \rightarrow Y$ is a homology equivalence.

Proof. Let $T=\{p \in \mathbb{P} \mid$ there exists $i \in \mathbb{N}$ such that $p$ divides some torsion coefficient of $\left.H_{i}(P ; \mathbb{Z})\right\}$.

In the first step we divide the collection of $C W$-complexes $\mathcal{W}$ into finitely many classes such that if $X$ and $Y$ belong to the same class then the map $f_{X Y}=d_{Y} u_{X}: X \rightarrow Y$ is a $\mathbb{Z} / p$-homology equivalence for all $p \in T$.

To do this, observe that all the groups $H_{i}(P ; \mathbb{Z} / p)$ are finite, hence, for each $i \in \mathbb{N}$ and $p \in \mathbb{P}$, there are only finitely many possible images of $H_{i, p}\left(u_{X}\right)$ in $H_{i}(P ; \mathbb{Z} / p)$. Thus, let $X$ and $Y$ belong to the same subclass $\mathcal{W}^{\prime}$ of $\mathcal{W}$ if and only if im $H_{i, p}\left(u_{X}\right)=\operatorname{im} H_{i, p}\left(u_{Y}\right)$ in $H_{i}(P ; \mathbb{Z} / p)$ for all $i \in \mathbb{N}$ and all $p \in T$.

In the second step we apply Lemma 1 to each class $\mathcal{W}^{\prime}$ as follows: we divide it into finitely many new classes such that if $X$ and $Y$ belong to the same class then there exists a finite sequence $\left\{X_{j}\right\}_{j=0}^{m}, m \geq 1$, where $X_{0}=X$, $X_{m}=Y, X_{j} \in \mathcal{W}^{\prime}$, such that the map $g_{X Y}=d_{Y} k_{X_{m-1}} k_{X_{m-2}} \ldots k_{X_{2}} k_{X_{1}} u_{X}$ : $X \rightarrow Y$ is a $\mathbb{Q}$-homology equivalence. By Lemma $2, g_{X Y}$ is a $\mathbb{Z} / p$-homology equivalence for all $p \notin T$.

Since im $H_{i, p}\left(u_{X_{j}}\right)$ are the same for each prime $p \in T$ and $X_{j} \in \mathcal{W}^{\prime}$ for all $j=0, \ldots, m$, it follows that $g_{X Y}$ is also a $\mathbb{Z} / p$-homology equivalence for all $p \in T$.

Thus $g_{X Y}$ is a $\mathbb{Q}$-homology equivalence and $\mathbb{Z} / p$-homology equivalence for all $p \in \mathbb{P}$. Applying Lemma 3 we conclude that $g_{X Y}$ is a homology equivalence. This ends the proof. 
Thus we can formulate

Theorem 1. Let $P$ be a polyhedron. All the $C W$-complexes dominated by $P$ can be partitioned into finitely many classes such that for any two $C W$-complexes belonging to the same class, there is a homology equivalence between them.

Proof. This is an immediate consequence of Lemma 4.

In what follows, $\widetilde{X}$ will denote, as usual, the universal covering space of $X$.

If $f: X \rightarrow Y$ is a cellular map of $C W$-complexes such that $f(x)=y$ for some vertices $x \in X, y \in Y$, then for any chosen $\widetilde{x} \in p^{-1}(x)$ and $\widetilde{y} \in p^{-1}(y)$ (where $p$ denotes the projections), there exists a unique map $\widetilde{f}: \widetilde{X} \rightarrow \widetilde{Y}$ such that $p \widetilde{f}=f p$ and $\widetilde{f}(\widetilde{x})=\widetilde{y}$. Then $\widetilde{f}$ induces homomorphisms $H_{i}(\widetilde{f})$ : $H_{i}(\widetilde{X}) \rightarrow H_{i}(\widetilde{Y})$ for $i \in \mathbb{N}$ (see [Hl, p. 107]).

In what follows, according to the remarks in the introduction, we consider pointed dominations and the pointed version of the Borsuk question. For simplicity, in our notations the base points will be omitted.

Let us prove the following

TheOREM 2. Let $P$ be a polyhedron with finite fundamental group $\pi_{1}(P)$. There are only finitely many homotopy types of $C W$-complexes dominated by $P$.

Proof. Consider all the $C W$-complexes $X \leq P$, each with a fixed domination $d_{X}: P \rightarrow X$ and inverse map $u_{X}: X \rightarrow P$, where $P$ is a polyhedron with finite fundamental group $\pi_{1}(P)$. Note that the universal covering space $\widetilde{P}$ of $P$ is a polyhedron.

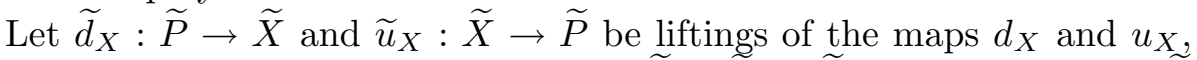
respectively, to the universal covers. Then $\widetilde{d}_{X}: \widetilde{P} \rightarrow \widetilde{X}$ is a domination of $\widetilde{P}$ over $\widetilde{X}$ with inverse map $\widetilde{u}_{X}$, and $\widetilde{k}_{X}=\widetilde{u}_{X} \widetilde{d}_{X}: \widetilde{P} \rightarrow \widetilde{P}$ is a homotopy idempotent. From now on, writing $\widetilde{X} \leq \widetilde{P}$, we will have in mind the domination $\widetilde{d}_{X}$ of $\widetilde{P}$ over $\widetilde{X}$.

The group $\pi_{1}(P)$ is finite, thus all the $C W$-complexes dominated by $P$ can be partitioned into finitely many classes such that $X$ and $Y$ belong to the same class if and only if $\operatorname{im} \pi_{1}\left(u_{X}\right)=\operatorname{im} \pi_{1}\left(u_{Y}\right)$, where $\pi_{1}\left(u_{X}\right): \pi_{1}(X) \rightarrow$ $\pi_{1}(P)$ and $\pi_{1}\left(u_{Y}\right): \pi_{1}(Y) \rightarrow \pi_{1}(P)$ are the homomorphisms induced by $u_{X}$ and $u_{Y}$, respectively.

Let $\mathcal{W}$ be a fixed class of this partition. Apply Lemma 4 to $\widetilde{P}$ and to the class of all $C W$-complexes $\widetilde{X} \leq \widetilde{P}$ for $X \in \mathcal{W}$. Thus we divide $\mathcal{W}$ into finitely many classes such that if $X$ and $Y$ belong to the same class, then there exists a finite sequence $\left\{X_{j}\right\}_{j=0}^{m}, m \geq 1$, where $X_{0}=X, X_{m}=Y$, 
$X_{j} \in \mathcal{W}$, such that the map $\widetilde{g}_{X Y}=\widetilde{d}_{Y} \widetilde{k}_{X_{m-1}} \widetilde{k}_{X_{m-2}} \ldots \widetilde{k}_{X_{2}} \widetilde{k}_{X_{1}} \widetilde{u}_{X}: \widetilde{X} \rightarrow \widetilde{Y}$ is a homology equivalence.

Since $\operatorname{im} \pi_{1}\left(u_{X_{j}}\right)$ in $\pi_{1}(P)$ are the same for all the $X_{j}$ (where $j=$ $0,1, \ldots, m)$, the map $g_{X Y}=d_{Y} k_{X_{m-1}} k_{X_{m-2}} \ldots k_{X_{2}} k_{X_{1}} u_{X}: X \rightarrow Y$ induces an isomorphism $\pi_{1}\left(g_{X Y}\right): \pi_{1}(X) \rightarrow \pi_{1}(Y)$.

By the Whitehead Theorem (see, for example, [Hi, Theorem 3.1, p. 107]), if $X$ and $Y$ are $C W$-complexes and there exists a map $f: X \rightarrow Y$ such that $f$ induces an isomorphism $\pi_{1}(f): \pi_{1}(X) \rightarrow \pi_{1}(Y)$ and isomorphisms $H_{i}(\widetilde{f}): H_{i}(\widetilde{X} ; \mathbb{Z}) \rightarrow H_{i}(\tilde{Y} ; \mathbb{Z})$ for all $i \in \mathbb{N}$, then $f$ is a homotopy equivalence. Thus the proof is finished.

Remark 1. The result of M. Putcha (Lemma 1.6 of [Pu2], see the Lemma in the Preliminaries) was used by L. Renner to prove that for a simply connected $C W$-complex $Z$ for which the rational cohomology algebra $H^{*}(Z ; \mathbb{Q})$ is finitely generated, there are only finitely many rational homotopy classes of rational retracts of $Z$ [Re]. However, neither this fact nor its proof in $[\mathrm{Re}]$ allow one to obtain the result of our paper. Moreover, our Corollary in the Preliminaries also enables a shorter and simpler proof of the result mentioned above.

REMARK 2. By our previous paper [K1], there exist polyhedra with nilpotent fundamental group dominating infinitely many different homotopy types (see the introduction and notice that every finitely generated, nilpotent torsion-free group is a poly-ZZ-group). However, applying Theorem 1 of the present paper we can show that every nilpotent polyhedron (i.e. a polyhedron with nilpotent fundamental group acting nilpotently on the higher homotopy groups) dominates only finitely many different homotopy types.

Acknowledgments. The author would like to thank Prof. Sławomir Nowak for bringing up the problem, for some conversations in 1990 which had an influence on obtaining the results of this paper, and for reading the manuscript.

\section{References}

[B1] K. Borsuk, On several problems of the theory of shape, in: Studies in Topology, Academic Press, 1975, 67-79.

[B2] - Some problems in the theory of shape of compacta, Russian Math. Surveys 34 (1979), no. 6, 24-26.

[B3] - Theory of Shape, Polish Sci. Publ., Warszawa, 1975.

[D] J. Dydak, Pointed and unpointed shape and pro-homotopy, Fund. Math. 107 (1980), 58-69.

[DS] J. Dydak and J. Segal, Shape Theory: An Introduction, Lecture Notes in Math. 688, Springer, Berlin, 1978. 
[HaHe] H. M. Hastings and A. Heller, Homotopy idempotents on finite-dimensional complexes split, Proc. Amer. Math. Soc. 85 (1982), 619-622.

[Hi] P. Hilton, An Introduction to Homotopy Theory, Cambridge Univ. Press, 1961.

[HMR] P. J. Hilton, G. Mislin and J. Roitberg, Localization of Nilpotent Groups and Spaces, North-Holland, 1975.

[Ho] W. Holsztyński, A remark on homotopy and category domination, Michigan Math. J. 18 (1971), 409.

[K1] D. Kołodziejczyk, Homotopy dominations within polyhedra, Fund. Math. 178 (2003), 189-202.

[K2] - Simply-connected polyhedra dominate only finitely many different shapes, Topology Appl. 112 (2001), 289-295.

[K3] -, There exists a polyhedron dominating infinitely many different homotopy types, Fund. Math. 151 (1996), 39-46.

[K4] - There exists a polyhedron with infinitely many left neighbors, Proc. Amer. Math. Soc. 129 (2001), 303-309.

[MS] S. Mardešić and J. Segal, Shape Theory. The Inverse System Approach, NorthHolland Math. Library 26, North-Holland, Amsterdam, 1982.

[Ma] M. Mather, Counting homotopy types of manifolds, Topology 4 (1965), 93-94.

[MP] M. A. Moron and F. R. Ruiz del Portal, Counting shape and homotopy types among fundamental absolute retracts: An elementary approach, Manuscripta Math. 79 (1993), 411-414.

[Pu1] M. S. Putcha, Linear Algebraic Monoids, Cambridge Univ. Press, 1988.

[Pu2] -, On linear algebraic subgroups, Trans. Amer. Math. Soc. 259 (1980), 457-469.

[Re] L. E. Renner, The homotopy types of retracts of a fixed space, J. Pure Appl. Algebra 69 (1990), 295-299.

[Tr] A. Trybulec, On shapes of movable curves, Bull. Acad. Polon. Sci. Sér. Sci. Math. Astronom. Phys. 21 (1973), 723-733.

[Wa] C. T. C. Wall, Finiteness conditions for CW-complexes, Ann. of Math. 81 (1965), $56-69$.

[WhG] G. Whitehead, Elements of Homotopy Theory, Springer, New York, 1978.

Faculty of Mathematics and Informational Sciences

Warsaw University of Technology

Pl. Politechniki 1

00-661 Warszawa, Poland

E-mail: dkolodz@mimuw.edu.pl

Received 14 January 2003;

in revised form 17 November 2003 